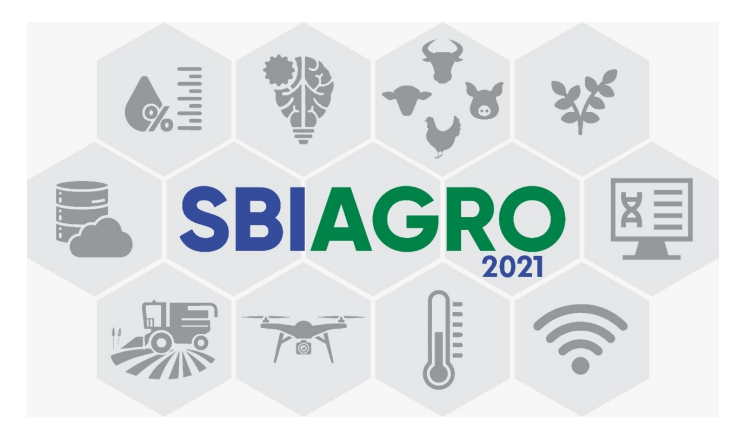

\title{
Implantação da Rede Temática GO-FAIR Agro Brasil: Primeiros Passos
}

\section{Debora P. Drucker ${ }^{1}$, Sérgio Manuel Serra da Cruz ${ }^{2,3}$, Antonio M. Saraiva ${ }^{4}$, Juliana}

M. Fortaleza ${ }^{5}$, Patrícia R. B. Bertin ${ }^{5}$, Victor P. M. Simão ${ }^{6}$, Milena A. Telles ${ }^{7}$, Alessandra R. da Silva ${ }^{8}$, Paulo S. S. Santos ${ }^{7}$, Carla G. N. Macario ${ }^{1}$

${ }^{1}$ Embrapa Agricultura Digital - Campinas - SP - Brasil

${ }^{2}$ Departamento de Computação, Universidade Federal Rural do Rio de Janeiro (UFRRJ) - Seropédica - RJ - Brasil

${ }^{3}$ Programa de Pós-Graduação em Informática, Universidade Federal do Rio de Janeiro (UFRJ) - Cidade Universitária, RJ - Brasil

${ }^{4}$ Departamento de Engenharia de Computação e Sistemas Digitais, Escola Politécnica,

Universidade de São Paulo (USP) - São Paulo, SP - Brasil

${ }^{5}$ Empresa Brasileira de Pesquisa Agropecuária - Secretaria de Desenvolvimento Institucional - Brasília, DF - Brasil

${ }^{6}$ Embrapa Meio Ambiente - Jaguariúna - SP - Brasil

${ }^{7}$ Empresa Brasileira de Pesquisa Agropecuária - Secretaria de Pesquisa e

Desenvolvimento - Brasília - DF - Brasil

${ }^{8}$ Empresa Brasileira de Pesquisa Agropecuária - Secretaria Geral - Brasília - DF - Brasil

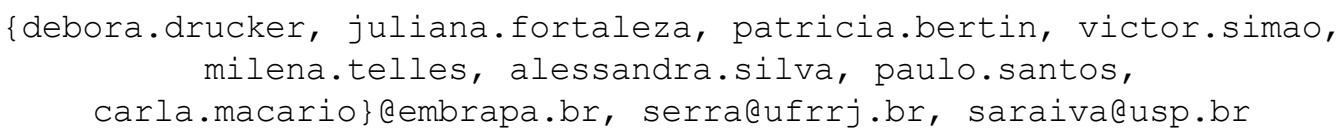

Abstract. This paper aims to present the current efforts to create the GO-FAIR Brazil Agro Thematic Implementation Network, highlighting its relationship with the GO-FAIR International and GO-FAIR Brazil initiatives. The main pillars are also highlighted, focusing on cultural change, training and infrastructure, as well as the proposed objectives of the Brazilian network, aimed at promoting the sharing and reuse of research data in agricultural sciences, supported by the FAIR principles. The results show the trajectory traveled so far and points to the elaboration of a Manifesto agreed upon and built through the participation of the communities of Agrarian Sciences and related areas. 


\begin{abstract}
Resumo. O presente trabalho tem o objetivo de apresentar os esforços que estão em curso para a estruturação da Rede de Implementação Temática GO-FAIR Brasil Agro, sua relação com as iniciativas GO-FAIR Internacional e GO-FAIR Brasil. A importância dos pilares de mudança cultural, treinamento e infraestrutura também é destacada, bem como a proposta de objetivos da rede brasileira voltada para promover o compartilhamento e o reúso de dados de pesquisa em ciências agrárias, apoiado nos princípios FAIR. Como resultados, o trabalho mostra a trajetória percorrida até o momento e aponta para a elaboração de um Manifesto pactuado e construído através de participação das comunidades de Ciências Agrárias e afins.
\end{abstract}

\title{
1. A Importância dos Princípios FAIR para as Ciências Agrárias
}

Dados são produtos valiosos da atividade de pesquisa [Heidorn, 2008] e boas práticas para sua gestão devem ser adotadas de modo a assegurar a confiabilidade e a reprodutibilidade do método científico e dos experimentos propriamente ditos. Nesse contexto, os princípios FAIR (Findable, Accessible, Interoperable, Reusable) acrônimo em inglês para dados "localizáveis, acessíveis, interoperáveis e reutilizáveis" [Wilkinson et al., 2016], vêm sendo amplamente adotados como norteadores da gestão de dados de pesquisa e viabilizadores de seu reúso.

Com o aumento exponencial da quantidade de dados passíveis de análise, a transição para uma ciência intensiva em dados vem acontecendo em todas as áreas do conhecimento [Hey et al., 2009]. A adoção dos princípios FAIR torna-se ainda mais relevante em áreas do conhecimento multi e interdisciplinares como as Ciências Agrárias $^{1}$ e a Agricultura Digital - aqui entendida como a inserção de tecnologias digitais em todas as fases da cadeia de valor tendo em vista a promoção de vantagens competitivas e benefícios socioambientais [Massruhá et al., 2020] - que integram conhecimentos sobre os meios físico, biológico, social e econômico. Assim, dados e objetos digitais utilizados para representar parâmetros de interesse podem variar em termos de formato, sintaxe e semântica, bem como em métodos de aquisição, tratamento e análise e em práticas de curadoria e de preservação. Por exemplo, são relevantes para a pesquisa e a tomada de decisão na agropecuária dados de sensores coletados em tempo real para monitorar variáveis de interesse agroclimático, dados coletados em sensores em máquinas agrícolas, dados de espécies em Áreas de Preservação Permanente (APPs), dados de experimentos de campo ou de entrevistas com produtores rurais.

No caso dos dados de sensores, aspectos relacionados com a qualidade e manutenção dos dispositivos em campo e com a transmissão, o armazenamento e o reúso adequados dos dados, bem como com os fluxos de análise e ajustes de modelos são preocupações determinantes para assegurar a qualidade dos experimentos e seus

\footnotetext{
1 A grande área do conhecimento Ciências Agrárias engloba Agronomia, Recursos Florestais e Engenharia Florestal, Engenharia Agrícola, Zootecnia, Medicina Veterinária, Recursos Pesqueiros e Engenharia de Pesca e Ciência e Tecnologia de Alimentos, conforme o diretório de grupos de pesquisa do $\mathrm{CNPq}$ - http://lattes.cnpq.br/web/dgp/ciencias-agrarias.
} 
resultados. Já no caso dos levantamentos de espécies, há um alto custo para sua obtenção em campo e para o tratamento de amostras que serão depositadas em coleções biológicas, cujos detalhes devem ser adequadamente registrados e os requisitos da Lei de Patrimônio Genético e Conhecimento Tradicional Associado (Lei no 13.123, de 20 de maio de 2015) obedecidos. Apenas posteriormente, com auxílio de especialistas, é possível associar um nome taxonômico aos espécimes coletados e, considerando que a taxonomia é também uma ciência viva, os nomes das espécies são atualizados ao longo do tempo e é preciso associar sinonímias para adequar a nomenclatura. Quando consideramos, ainda, dados de entrevistas com agricultores, muitas vezes os dados são oriundos de questionários semi-estruturados, trazendo a complexidade de tratar dados quanti e qualitativos e, do ponto de vista ético e de conformidade, é preciso atentar aos princípios da Lei Geral de Proteção de Dados Pessoais (LGPD - Lei no 13.709, de 14 de agosto de 2018).

Considerando a diversidade de aplicações dos princípios FAIR para as Ciências Agrárias, sua relevância do contexto brasileiro para a produção de alimentos, agronegócios e sua relação com a saúde humana e do ambiente, o presente trabalho tem o objetivo de apresentar a Rede de Implementação Temática GO-FAIR Brasil Agro: sua relação com as iniciativas GO-FAIR Internacional e GO-FAIR Brasil e sua proposta de estruturação.

\section{A Iniciativa GO-FAIR}

A rede GO-FAIR é uma iniciativa internacional iniciada em 2018 na Europa e atualmente presente em diversos países. Ela é dirigida pelas partes interessadas e autogovernada, e visa implementar os princípios FAIR, tornando os dados localizáveis, acessíveis, interoperáveis e reutilizáveis. A rede GO-FAIR conta com uma estrutura de governança, escritórios regionais e redes de implementação temáticas, sendo estruturado em três pilares: GO-CHANGE, que trata de mudança cultural em vários níveis de organização institucional na transição para a ciência aberta; GO-TRAIN, relacionado com a capacitação de recursos humanos para implementar os princípios FAIR nas diferentes etapas do ciclo de vida dos dados e GO-BUILD, no qual padrões e serviços de dados FAIR são desenvolvidos e implementados ${ }^{2}$. Atualmente, a rede mantém estreita colaboração com o Committee on Data for Science and Technology do International Science Council (CODATA/ICSU) ${ }^{3}$ e outras instituições internacionais relevantes como a Research Data Alliance (RDA) ${ }^{4}$ e o World Data Systems (WDS) ${ }^{5}$, que têm compromisso de trabalhar juntas para otimizar o ecossistema global de dados e identificar as oportunidades e necessidades que irão acionar as infraestruturas federadas para atender à nova realidade da ciência intensiva em dados.

\footnotetext{
${ }^{2}$ Mais detalhes podem ser encontrados em https://www.go-fair.org/go-fair-initiative.

${ }^{3}$ Informações sobre o Comitê podem ser encontradas em https://codata.org/.

${ }^{4} \mathrm{O}$ portal da https://rd-alliance.org/acomoda detalhes sobre as iniciativas em andamento e detalhes dos grupos de trabalho e grupos de interesse.

${ }^{5}$ Mais informações sobre a WDS estão disponíveis em https://www.worlddatasystem.org/.
} 
Há escritórios regionais da rede GO-FAIR em diversos países, como Alemanha, Áustria, Brasil, China, Dinamarca, França, Estados Unidos e Holanda, os quais são articulados pelo escritório de coordenação "GO-FAIR International Support and Coordination Office (GFISCO)", que promove a troca de experiências para avanço global da implementação dos princípios FAIR. Além disso, há dezenas de Redes de Implementação Temáticas no âmbito internacional (INs, acrônimo para Implementation Networks), que se comprometem a implementar os princípios FAIR para dados e serviços dentro dos três pilares: GO-BUILD (Tecnologia), GO-CHANGE (Cultura) e GO-TRAIN (Treinamento). Algumas INs estão relacionadas com domínios do conhecimento específicos, como Saúde e Biodiversidade, enquanto outras são aplicáveis a todos os domínios, como a IN para Centros de Competência em Custódia de Dados (tradução livre para "Data Stewardship Competence Centers" - DSCC). Há, ainda, iniciativas voltadas para desafios da atualidade, como a IN "Virus Outbreak Data Network (VODAN)", formada no início da pandemia de COVID-19 e que envolve diversos países, tendo contribuído com as necessidades globais de mobilização de dados para enfrentar os desafios que se apresentaram de forma abrupta para a humanidade e reforçando a importância da consonância com os princípios FAIR.

Diversas instituições brasileiras estão representadas nas INs existentes e as atividades do escritório regional GO-FAIR Brasil são articuladas com as iniciativas globais, promovendo o intercâmbio de conhecimentos e a troca de experiências. A seguir, descreveremos o escritório brasileiro em mais detalhes.

\section{O Escritório Regional GO-FAIR Brasil}

O Escritório do GO-FAIR Brasil foi criado em 2018, durante o evento de 20 anos da Rede Scientific Electronic Library Online (SciELO). Na ocasião, foi redigido e assinado o Manifesto da Rede GO-FAIR Brasil, coordenado pelo Instituto Brasileiro em Informação Científica e Tecnológica (IBICT) [Sales et al 2020]. Sua missão é:

"Promover, apoiar e facilitar a adoção dos princípios FAIR na gestão de dados no Brasil, contribuindo para ampliar a visibilidade dos saberes científicos, aprimorar a transparência e a reprodutibilidade da pesquisa, favorecer o reuso de dados e acelerar o

desenvolvimento científico em todas as áreas, através da gestão de dados por domínios."

\footnotetext{
${ }^{6} \mathrm{O}$ portal https://www.go-fair-brasil.org/ contém as informações gerais da rede.
} 
O funcionamento da iniciativa GO-FAIR está descrito em sua Carta de Princípios $^{7}$, na qual constam também os objetivos, princípios e estrutura de governança da rede. Analogamente ao GO-FAIR internacional, a rede brasileira também se organiza em três pilares e em Redes de Implementação Temáticas, as quais contribuem para a disseminação e a adoção dos princípios FAIR no Brasil em diferentes domínios do conhecimento.

A criação de uma nova Rede de Implementação Temática (RI) se dá pela representação de instituições-chave no referido domínio, que engaja participantes a escrever e divulgar um Manifesto em evento significativo da área. Atualmente, a rede brasileira conta as Redes de Implementação Temáticas, em diferentes níveis de maturidade, nas áreas da Saúde e a sub-rede Enfermagem; Humanidades; Ciências Nucleares; Ensino, Ciência, Tecnologia e Inovação; Biodiversidade e Agro, a qual será descrita na próxima seção. A Rede brasileira promove encontros para a troca de experiências entre RIs e incentiva trabalhos conjuntos no âmbito de seus pilares, ou seja, promoção de treinamento em conteúdos em comum para diferentes domínios, ações de disseminação e promoção de mudança cultural e atividades voltadas à implantação de soluções tecnológicas para a adoção de padrões de dados e objetos digitais, em consonância com os esforços globais de implementação dos princípios FAIR.

O escritório brasileiro atua também em sincronia com os demais escritórios regionais distribuídos pelo mundo e participa ativamente de ações globais nessa temática, como a participação no FairFestival ${ }^{8}$, que ocorreu em junho de 2021, de forma totalmente virtual, e contou com apresentações de vários membros da rede brasileira.

\section{A Rede de Implementação Temática GO-FAIR Brasil Agro}

A Empresa Brasileira de Pesquisa Agropecuária - Embrapa foi convidada pela Coordenação da Rede GO-FAIR Brasil para liderar a implementação da Rede Temática em pesquisa agropecuária, a qual foi denominada "GO-FAIR Agro". O Centro de pesquisas em Informática da empresa, Embrapa Agricultura Digital, por sua vocação em atuação na área de Computação, foi designado para coordenar as atividades da Embrapa na Rede, agregando representantes de outros centros da Embrapa. Além da coordenação da Embrapa, representantes de outras instituições relevantes às Ciências Agrárias foram convidados a compor o colegiado de gestão da RI GO-FAIR Brasil Agro: Universidade de São Paulo (USP) e Universidade Federal Rural do Rio de Janeiro (UFRRJ). Essa é uma composição inicial e espera-se que o colegiado de gestão seja ampliado ao longo do tempo, agregando a representatividade do universo produtor e consumidor de dados das ciências agrárias com a participação de outras organizações.

\footnotetext{
7 A Carta de Princípios da Rede Go-FAIR Brasil está disponível em $\frac{\text { https://www.go-fair-brasil.org/documentos }}{8 \text { As gravações do evento e documentos associados estão disponíveis em }}$ https://www.go-fair.org/events/fair-festival-2021/.
} 
O esboço do Manifesto da RI GO-FAIR Brasil Agro será apresentado no XIII Congresso Brasileiro de Agroinformática (SBIAgro), em 2021. A proposta inicial, a ser discutida em evento representativo da comunidade (conforme orientação da rede GO-FAIR), é que a missão da Rede GO-FAIR Brasil Agro seja "promover o compartilhamento e o reúso de dados de pesquisa (tanto por humanos quanto por máquinas) em Ciências Agrárias, apoiado nos princípios FAIR". Os objetivos específicos propostos são:

1. Promover pesquisas e inovações sobre:

a. repositórios de dados de pesquisa nacionais e internacionais que poderão armazenar e compartilhar, de forma confiável, os dados de pesquisa em ciências agrárias;

b. padrões de metadados e proveniência de dados específicos;

c. padrões de interoperabilidade tecnológica e semântica dos dados;

d. modelos de plano de gestão de dados.

2. Desenvolver metodologias voltadas para as práticas dos produtos e serviços FAIR, que atendam às necessidades disciplinares e operacionais da área das Ciências Agrárias.

3. Promover difusão do conhecimento através de encontros, cursos, workshops e seminários visando impulsionar e disseminar os princípios FAIR entre os membros da Rede GO-FAIR Brasil Agro e sociedade em geral.

4. Trabalhar de forma articulada e colaborativa com a Rede GO-FAIR Brasil e Redes de Implementação Temáticas Nacionais e com a Rede de Implementação internacional Food Systems.

O texto do Manifesto será trabalhado conjuntamente com a comunidade durante o XIII SBIAgro e a primeira versão do documento será produzida. Outros atores relevantes, tanto produtores quanto consumidores de dados, ainda não representados, serão convidados a integrar à rede e as etapas de implementação da RI GO-FAIR Brasil Agro serão construídas conjuntamente com a comunidade, em consonância com a Carta de Princípios da Rede GO-FAIR Brasil.

Ainda que os princípios FAIR estejam bem estabelecidos em termos conceituais, quando colocado em prática, geram dúvidas e incertezas. A rápida adoção internacional dos princípios - por instituições de pesquisa, universidades, editores, gestores de dados e de infraestrutura de dados e agências de fomento - levou a uma ambiguidade e a uma ampla interpretação do estado "FAIRness" dos dados e repositórios [Wilkinson et al., 2018], uma vez que os princípios são tecnologicamente agnósticos e não definem estritamente como alcançar esse estado, mas descrevem um conjunto de características, atributos e comportamentos que aproximam um objeto digital desse objetivo. Como resultado, várias abordagens, metodologias, workflows e métricas para a "FAIRificação" de dados e objetos digitais foram desenvolvidas [Chue Hong et al., 2021], [Cox et al. 2021], [Jacobsen et al., 2020], [RDA FAIR Data Maturity Model Working Group, 2020], porém, não necessariamente funcionais para todas as áreas do conhecimento, devido à complexidade e heterogeneidade dos dados e às especificidades das áreas de conhecimento. 
Algumas iniciativas internacionais no contexto da agricultura digital já vem recomendando práticas mais efetivas de gestão de dados com a adoção dos Princípios FAIR, por exemplo na Plataform for Big Data in Agriculture (CGIAR ${ }^{9}$ ), no Global Open Data for Agriculture \& Nutrition $\left(\right.$ GODAN $\left.^{10}\right)$, no Centre for Agriculture and Bioscience International $\left(\mathrm{CABI}^{11}\right)$, entre outros. No Brasil, esses esforços ainda são incipientes. Destacamos que operacionalizar os Princípios FAIR em sistemas e repositórios em um país continental e com tamanha abrangência e importância das cadeias do agronegócio é uma tarefa árdua em função do seu alto grau de subjetividade e complexidade, especialmente nas Ciências Agrárias, pois, para produzir mais safras com menos recursos e, ao mesmo tempo, mitigar os efeitos negativos sobre a sociedade e o meio ambiente, é preciso abordagens interdisciplinares intensivas nas análises de dados [Drucker et al., 2021]. A troca de dados entre os diferentes domínios - planta, animal, meio ambiente, ciências sociais, dentre outros - é um grande desafio, ainda maior no contexto da agricultura tropical.

Esforços para a disseminação e adoção dos princípios FAIR têm sido desenvolvidos em todo o mundo, por meio de redes de escritórios regionais, estudos e novas definições de padrões de dados e metadados; disseminação do uso de proveniência de dados, vocabulários controlados, ontologias e adoção de identificadores persistentes que proporcionam significado preciso aos dados e aos demais objetos a eles vinculados [Henning et al., 2019]. Espera-se que, com a implementação da Rede GO-FAIR Brasil Agro, pesquisadores e profissionais que permeiam toda a cadeia do agronegócio e que atuam na interface entre as Ciências Agrárias e da Ciência da Computação contribuam para alavancar a adoção dos princípios FAIR neste domínio científico, bem como para expandir a cultura da promoção de melhores práticas de gestão de dados e de outros objetos digitais. Além disso, o engajamento da comunidade proporciona novas ideias e colaborações entre indivíduos e instituições, podendo expandir para uma efetiva cooperaçãocom a IN Food Systems e outras redes com as quais há interesses comuns, como, por exemplo, a IN BiodiFAIRse e a RI Nacional Biodiversidade. Adicionalmente, avanços no sentido da adoção dos princípios FAIR em ações que independem de domínio devem ser promovidos pela atuação com as demais RIs da rede brasileira, bem como com as iniciativas dos três pilares para promover a mudança cultural, a capacitação de pessoas e o desenvolvimento tecnológico.

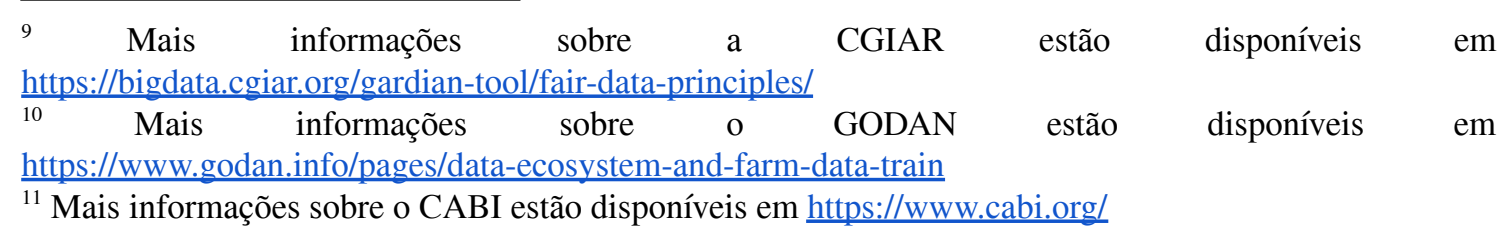




\section{References}

Chue Hong, N. P., Katz, D. S., Barker, M., Lamprecht, A.-L., Martinez, C., Psomopoulos, F. E., Harrow, J., Castro, L. J., Gruenpeter, M., Martinez, P. A., Honeyman, T., et al. (2021). FAIR Principles for Research Software (FAIR4RS Principles). Research Data Alliance. DOI: 10.15497/RDA00065.

Cox, S.J.D.; Gonzalez-Beltran A.N; Magagna B.; Marinescu M.-C. (2021). Ten simple rules for making a vocabulary FAIR. PLoS Comput Biol17(6): e1009041. https://doi.org/10.1371/journal.pcbi.1009041.

Drucker, D. P.; Fortaleza, J. M.; Bertin, P. R. B.; Vacari, I.; Macario, C. G. do N. (2021). Rumo à rede de implantação GO FAIR 'Agro' Brasil: a experiência de uma organização de PD\&I na implantação dos princípios FAIR In: Sales, L. F.; Veiga, V. S. O.; Henning, P.; Sayão, L. F. (Ed.). Princípios FAIR aplicados à gestão de dados de pesquisa. Rio de Janeiro, RJ: IBICT. cap. 3. p. 53-68.

Jacobsen, A. et al. (2020). A generic workflow for the data FAIRification process. Data Intelligence 2, 56-65. https://doi: 10.1162/dint_a_00028.

Henning, P. et al. 2019. Desmistificando os princípios fair: conceitos, métricas, tecnologias e aplicações inseridas no ecossistema dos dados FAIR. Pesquisa Brasileira em Ciência da Informação e Biblioteconomia, Paraíba, v. 14, n. 3, p. 175-192. 10.22478/ufpb.1981-0695.2019v14n3.46969.

HEY, Tony; TANSLEY, Stewart; TOLLE, Kristin, (ed.). 2009. The fourth paradigm: data-intensive scientific discovery. Redmond: Microsoft Research. Disponível em: https://www.microsoft.com/en-us/research/wp-content/uploads/2009/10/Fourth_Para digm.pdf. Acesso em 1 ago 2021.

Massruhá, S. M. F. S.; Leite, M. A. De A.; Luchiari Junior, A.; Evangelista, S. R. M. (2020). A transformação digital no campo rumo à agricultura sustentável e inteligente. In: Massruhá, S. M. F. S.; Leite, M. A. De A.; Oliveira, S. R. De M.; Meira, C. A. A.; Luchiari Junior, A.; Bolfe, E. L. (Ed.). Agricultura digital: pesquisa, desenvolvimento e inovação nas cadeias produtivas. Brasília, DF: Embrapa. cap. 1, p. 20-45.

RDA FAIR Data Maturity Model Working Group (2020). FAIR Data Maturity Model: specification and guidelines. Research Data Alliance. DOI: 10.15497/RDA00050

Sales, L.; Henning, P.; Veiga, V.; Costa, M.M.; Sayão, L.F.; Santos, L.O.B.S.; Pires, L.F. (2020) GO FAIR Brazil: A Challenge for Brazilian Data Science. Data Intelligence 2 (1-2): 238-245. https://doi.org/10.1162/dint a 00046.

Wilkinson, M., Dumontier, M., Aalbersberg, I. et al. (2016). The FAIR Guiding Principles for scientific data management and stewardship. Sci Data 3, 160018. https://doi.org/10.1038/sdata.2016.18.

Wilkinson, M.D. et al. (2018). Comment: A design framework and exemplar metrics for FAIRness. Scientific Data 5, 1-4. doi: 10.1038/sdata.2018.118. 